\title{
6.3 DYNAMICAL STABILITY OF NEUTRON STARS
}

\author{
G. CHANMUGAM and M. GABRIEL \\ Institut d'Astrophysique, Université de Liege, B4200 Cointe-Ougree, Belgium
}

\begin{abstract}
The Nemeth-Sprung equation of state is modified and used to obtain neutron star models. Contrary to the results of some authors it is found that neutron stars with central densities $\lesssim 10^{14} \mathrm{~g} \mathrm{~cm}^{-3}$ are dynamically stable. It is suggested that some pulsars may belong to this category of stars.
\end{abstract}

\section{Introduction}

Neutron star models determined as accurately as possible are most valuable for understanding the behaviour of pulsars. The equation of state of neutron star matter at densities greater than about $5 \times 10^{14} \mathrm{~g} \mathrm{~cm}^{-3}$ is poorly known. Fortunately however, there has been much progress recently in the theory of nuclear matter so that our understanding of neutron star matter at densities less than about $5 \times 10^{14} \mathrm{~g} \mathrm{~cm}^{-3}$ should be better than when some of the earlier models on neutron stars were constructed (Tsuruta, 1964, Meltzer and Thorne, 1966). In this work we use the NemethSprung (1968) equation of state, which we modify, in order to discuss neutron star models and their stability.

\section{Equation of State}

Nemeth and Sprung have used Brueckner theory to derive the equation of state of neutron star matter consisting of electrons, protons, neutrons and muons. In their work they use the soft core Reid (1967) potential which is more recent than the Levinger and Simmons potentials (1961) used in constructing neutron star models (Tsuruta, 1964, Cohen et al., 1970). Nemeth and Sprung (1968) have calculated four equations of state (their results contain some errors which we have corrected) of which they believe that their equation of state $(2 b)$ is the best one. It is this equation of state that we use here. Furthermore, since their equation of state does not include the effects of nuclear clustering we have included these effects, using the binding energy formula of Green (1954) and essentially following the methods of Langer et al. (1969).

We find for increasing values of $\varrho \gtrsim 3 \times 10^{11} \mathrm{~g} \mathrm{~cm}^{-3}$ that the system consists of electrons, neutrons and nuclei. The nuclei becoming increasingly neutron rich as $\varrho$ increases. At $\varrho \approx 4.6 \times 10^{13} \mathrm{~g} \mathrm{~cm}^{-3}$, protons appear while the nuclei disappear at $\varrho \approx 4.7 \times 10^{13} \mathrm{~g} \mathrm{~cm}^{-3}$. Muons appear at $\varrho \approx 1.8 \times 10^{14} \mathrm{~g} \mathrm{~cm}^{-3}$. These thresholds are in essential agreement with the results of Langer et al. except that we find that the phase with electrons, protons, neutrons and nuclei exists over a narrower density range. At low densities the effects of nuclear forces are small and hence the difference between our equation of state and that of Langer et al. becomes negligible (Figure 1). It was also found that when nuclear clustering is included, the Nemeth-Sprung equation of state gives higher pressures than when clustering is not included. This is because the additional protons in the nuclei, when nuclear clustering is included, 
have to be neutralised by additional electrons which contribute significantly to the pressure, particularly at low densities. The adiabatic index is however smaller when nuclear clustering is included. It is important to realize that the treatment of the phase with nuclei leaves much to desired. Coulomb interactions, lattice effects, shell model effects and effects of the neutron sea on the nuclei have been neglected in this calculation. Electromagnetic many body forces, which have been suggested to be of possible

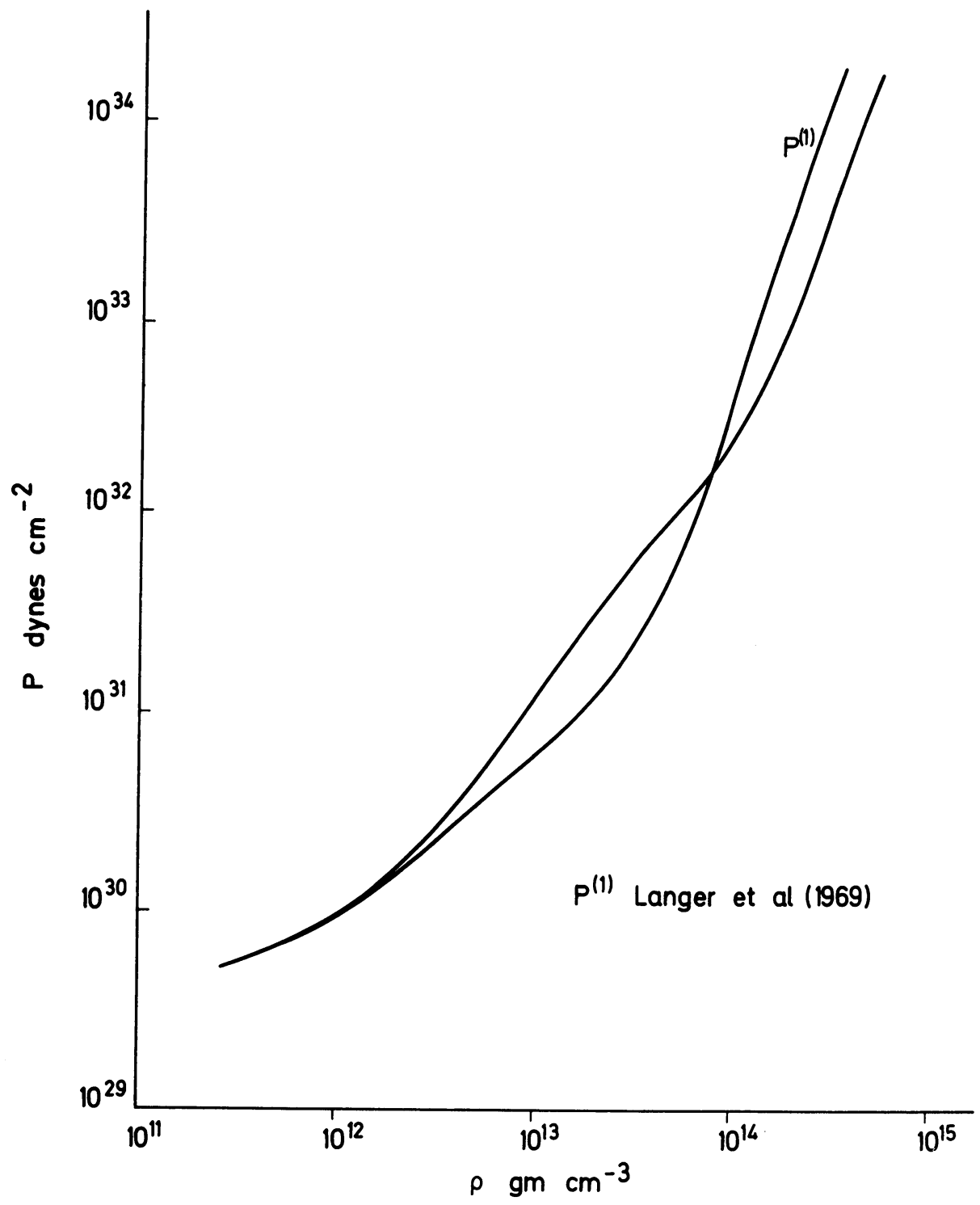

Fig. 1. Pressure as a function of density, from the modified Nemeth-Sprung equation of state, compared with that from the results of Langer et al. (1969). 
importance in dense electron gases as found in neutron stars (Chanmugam and Schweber, 1970), have also been neglected.

\section{Neutron Star Models}

We have used the modified Nemeth-Sprung equation of state to integrate the general relativistic stellar structure equations to obtain neutron star models (Table I) with central densities $\varrho_{c}$ up to $4.8 \times 10^{14} \mathrm{~g} \mathrm{~cm}^{-3}$. The models have a minimum mass of $\approx 0.07 M_{\odot}$ at $\varrho_{c} \approx 2.4 \times 10^{14} \mathrm{~g} \mathrm{~cm}^{-3}$. For $\varrho_{c} \gtrsim 2.4 \times 10^{14} \mathrm{~g} \mathrm{~cm}^{-3}$ we find that the masses of neutron stars are smaller than generally believed earlier. In particular our models have masses about one fifth of those given by Cohen et al. (1970). The recent models of Wang et al. (1970) also have small masses. We find that our models have radii in rough agreement with the results of Cohen et al. but are much smaller than those of Wang et al. This is probably because the masses of these neutron stars are sensitive to the equation of state at high densities whereas the radii are sensitive to the equation of state at low densities. The periods of radial oscillation and the thickness of the crystalline layer are in reasonable agreement with results of Cohen et al.

TABLE I

Neutron star models

\begin{tabular}{llc}
\hline Central density in $\mathrm{g} \mathrm{cm}^{-3}$ & Mass in solar units & Radius in $\mathrm{km}$ \\
\hline $4.77 \times 10^{14}$ & 0.23 & 10.6 \\
$3.92 \times 10^{14}$ & 0.16 & 12.0 \\
$3.17 \times 10^{14}$ & 0.11 & 14.9 \\
$2.52 \times 10^{14}$ & 0.08 & 53.2 \\
$1.98 \times 10^{14}$ & 0.83 & 3580.0 \\
$1.51 \times 10^{14}$ & 0.80 & 1415.8 \\
$5.8 \times 10^{13}$ & 0.74 & 1057.3 \\
$4.35 \times 10^{13}$ & 0.73 & 1030.7 \\
$2.71 \times 10^{13}$ & 0.71 & 930.6 \\
$8.24 \times 10^{12}$ & 0.70 & 711.1 \\
\hline
\end{tabular}

Cohen et al. and Wang et al. find that their models have a minimum mass at $\varrho_{c} \approx 10^{14} \mathrm{~g} \mathrm{~cm}^{-3}$. They also find that their models with central densities $\varrho_{c} \lesssim 10^{14} \mathrm{~g} \mathrm{~cm}^{-3}$ are unstable. In their calculations they use the adiabatic index along the equation of state $\gamma_{E}$ to determine the criteria for dynamical stability. However, the period of oscillations of neutron stars are in general much smaller than the nuclear reaction rates (Meltzer and Thorne, 1966) so that one should use the adiabatic index at constant composition $\gamma_{c}$ in determining the criterion for stability. We find that $\gamma_{c}$ is sufficiently large (Figure 2) so that the models with $10^{13} \lesssim \varrho_{c} \lesssim 10^{14} \mathrm{~g} \mathrm{~cm}^{-3}$ are dynamically stable. These models have masses $\approx 0.75 M_{\odot}$ and radii $\approx 1000 \mathrm{~km}$ (Table I). For $\varrho_{c} \lesssim 10^{13} \mathrm{~g} \mathrm{~cm}^{-3}$ it is possible to have long periods of the order of the nuclear reaction rates so that the $\gamma$ to be used is amplitude and period dependent and lies between $\gamma_{E}$ and $\gamma_{c}$, and the problem becomes more complicated. 


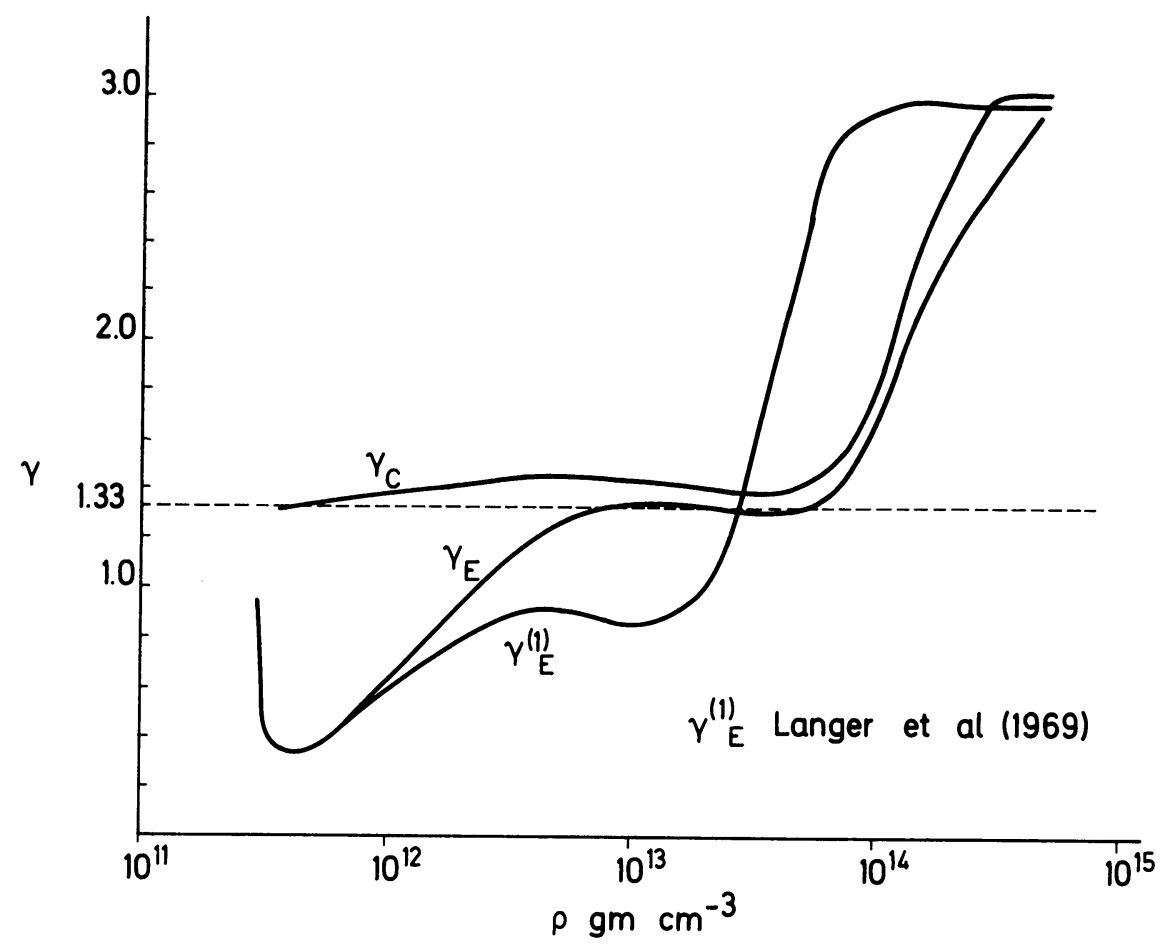

Fig. 2. The adiabatic indices $\gamma_{E}$ (along the equation of state) and $\gamma_{c}$ (at constant composition) from the modified Nemeth-Sprung equation of state, compared with $\gamma_{E}$ from the results of Langer et al. (1969).

\section{Application to Pulsars}

If we assume that the Crab pulsar is a rotating neutron star we find for our models with $\varrho_{c} \gtrsim 2.4 \times 10^{14} \mathrm{~g} \mathrm{~cm}^{-3}$ that the rotational energy loss is insufficient to account for the $10^{38} \mathrm{erg} \mathrm{sec}^{-1}$ necessary to drive the Crab nebula. Despite the small masses and because of the large radii in their models Wang et al. do find sufficient rotational energy loss to drive the Crab nebula. However, it should be noted that Wang et al. use the equation of state of Langer $e t$ al. at low densities and do not explicitly calculate effects due to nuclear clustering. Since the potentials used by Wang et al. are different from that used by Langer et al. their equation of state at low densities is not self consistant. Hence similar criticisms apply to their values for radii. They also do not include effects due to the presence of protons, muons and electrons.

Ginzburg et al. (1969) have suggested that some pulsars may be rotating and vibrating, the period $\approx 1 \mathrm{sec}$ corresponding to rotation and the period $\gtrsim 10^{-2} \mathrm{sec}$ corresponding to vibration. This theory has been criticized on the grounds that neutron stars with periods of vibrations $\gtrsim 10^{-2}$ sec occur only over a very narrow range for models near the mass minimum. However this criticism applies only to 
models where $\gamma_{E}$ is used in discussing the stability. But if, as in our work, $\gamma_{c}$ is used, stars with periods $\gtrsim 10^{-2}$ sec occur over a wider range of density, adding support to the theory of Ginzburg et al.

In conclusion we would like to suggest that there exist stable neutron stars with central densities $\varrho_{c} \lesssim 10^{14} \mathrm{~g} \mathrm{~cm}^{-3}$, and it is likely that at least some pulsars belong to this category.

\section{Acknowledgements}

We would like to thank Prof. P. Ledoux for valuable discussions. One of us (G.C.) thanks the Université de Liège for a research fellowship.

\section{References}

Chanmugam, G. and Schweber, S. S.: 1970, Phys. Rev. A1, 1369.

Cohen, J. M., Langer, W. D., Rosen, L. C., and Cameron, A. G. W.: 1970, Astrophys. Space Sci. 6, 228.

Ginzburg, V. L., Zheleznyakov, V. V., and Zaitsev, V. V.: 1969, Astrophys. Space Sci. 4, 464.

Green, A. E. S.: 1954, Phys. Rev. 95, 1006.

Langer, W. D., Rosen, L. C., Cohen, J. M., and Cameron, A. G. W.: 1969, Astrophys. Space Sci. 5, 259.

Levinger, J. S. and Simmons, L. M.: 1961, Phys. Rev. 124, 916.

Meltzer, D. W. and Thorne, K. S.: 1966, Astrophys. J. 145, 514.

Nemeth, J., Sprung, D. W. L.: 1968, Phys. Rev. 176, 1496.

Reid, R. V., Jr.: 1967, Thesis, Cornell University.

Tsuruta, S.: 1964, Thesis, Columbia University.

Wang, C. G., Rose, W. K., and Schlenker, S. L.: 1970, Astrophys. J. 160, L17.

Discussion of this paper was deferred until after the following paper by Bonazzola. 